\title{
Loss of stability of open two-link mechanisms
}

\author{
Leonid Kondratenko ${ }^{1}$, Lubov Mironova ${ }^{2}$, Vladimir Dmitriev ${ }^{3}$ \\ ${ }^{1}$ Central Research Institute of Machine Building Technology, \\ Sharikopodshipnikovskaya Str., Moscow, 115088, Russia \\ ${ }^{2,3}$ Moscow Aviation Institute (State National Research University), \\ Volokolamskoe sh., Moscow, 125993, Russia \\ ${ }^{1}$ Corresponding author \\ E-mail: ${ }^{1}$ kondrat.leonid@yandex.ru, ${ }^{2}$ mironova_lub@mail.ru, ${ }^{3}$ vgd2105@mail.ru \\ Received 10 May 2019; accepted 21 May 2019 \\ DOI https://doi.org/10.21595/vp.2019.20789
}

Check for updates

Copyright $(C 2019$ Leonid Kondratenko, et al. This is an open access article distributed under the Creative Commons Attribution License, which permits unrestricted use, distribution, and reproduction in any medium, provided the original work is properly cited.

\begin{abstract}
The problems of the loss of stable operation of two-link mechanisms consisting of the driving and driven members connected by long force lines are considered. The problem was solved on the basis of a new approach to the study of the dynamics of these systems with straight rods, based on the use of the equations of momentum (for longitudinal oscillations) and angular momentum (for torsional oscillations) in differential form. Stability was estimated by the first Lyapunov method, which consists in solving the resulting differential equations. As a result of modeling the work of a volumetric hydraulic drive, modes of self-oscillations are revealed. The areas of stability and instability are determined.
\end{abstract}

Keywords: two-link mechanisms, force lines, distributed parameters, system stability, momentum, moment of momentum, straight rod, oscillation frequency, volume hydraulic drive.

\section{Introduction}

Two-link mechanical systems contain a leading (input) link-energy source (engine, pump) and a driven (output) link-executive body (cutting part of the borer, mill, chisel, etc.). These links are connected by a force line. Such systems are widely used in industry. Moreover, in a number of processes (drilling, drilling aperture, drilling wells), feedback is not used. Loss of stability leads to self-oscillations, when loads on system components increase significantly, results of work changes, a sudden stop can occur. Usually such modes are highly undesirable.

The problem of the stability of motion by mathematicians and mechanics has been studied since the 19th century. To solve such problems are used the criteria and theories of Routh, Gurwitz, Lyapunov, Chetaev, Mikhailov, Nyquist, Bolotin, Popov [1-6] and etc.

In a number of publications, such problems are solved by an approximate method with the replacement of the corresponding functional equations by suitable finite-dimensional difference schemes [7, 8]. In the general case, the conditions for the stable operation of a system with distributed parameters are considered in [9-11].

\section{Statement of the problem}

Usually, for solving such problems, the well-known wave equations or equations based on the Lagrange equations of the 2nd kind are used [11].

This approach allows us to estimate the change in the displacements of the elementary volume of the rod and other parameters in time and space. However, such information, if it is necessary to take into account the interrelationship of a large number of factors, is on the one hand redundant, since it is often enough to know under what conditions do self-oscillations arise of the rotation frequency of a technological object (executive body), i.e. stability is lost, and when what is not. On the other hand, due to the lack of information about the stresses developed in the dynamic process, it is difficult to estimate the probability of part failure or disruption to the work process.

In the stated approaches to solving problems, a linear dependence of the stress on the movement of particles in the material is assumed and a corresponding recalculation is made. 
However, this is not always justified, since it is known from rheology that the modulus of elasticity may depend on the oscillation frequency [12]. In addition, depending on the stresses, the stem of the gun drill or the drill string may bend and thereby change the characteristics of the formation of force factors on the executive body and the results of work of the mechanism. At the same time, various non-linear effects, including significant ones, have a great influence on the functioning of the system.

\section{Basic equations}

To solve assigned problem when considering longitudinal oscillations in a straight solid rod in the absence of mass forces, the equation of momentum in differential form was used and the equation of longitudinal vibrations $[11,13,14]$ :

$\rho \frac{\partial v}{\partial t}=-\frac{\partial \sigma}{\partial x}, \quad \frac{\partial^{2} u}{\partial t^{2}}=\frac{E}{\rho} \frac{\partial^{2} u}{\partial x^{2}}$

here $v$ is velocity of the longitudinal displacement of the point in the considered cross-section $(v=\partial u / \partial t) ; u$ is displacement along the $x$ axis; $\sigma$ is longitudinal (normal) stress; $\rho$ is density of the material; $E$ is elastic modulus; $t$ is time.

For $E=$ const, $\rho=$ const from Eqs. (1) and (2) we obtain the expression $\partial \sigma / \partial x=\left(E \partial^{2} u\right) /\left(\partial x^{2}\right)$, which, after integration with respect to $x$, and then differentiation with respect to $t$, is reduced to:

$\frac{1}{E} \frac{\partial \sigma_{x}}{\partial t}=-\frac{\partial v}{\partial x}$

Eqs. (1), (2), first derived in [13], make it possible to explicitly describe the oscillations of velocities and stresses in a rod. Torsional vibrations can be described in a similar way $[14,15]$. It should be noted that the process of motion transmission in systems with hydraulic lines is characterized by the equations [16]:

$\frac{\partial v}{\partial t}=-\frac{1}{\rho} \frac{\partial P}{\partial x}-2 \rho_{0} \tau_{p} r_{p}, \quad \frac{\partial P}{\partial t}=-\kappa \frac{\partial v}{\partial x}$

here $\rho_{0}$ is initial density of the medium; $P$ is line pressure; $\kappa$ is reduced modulus of elasticity of the highway; $\tau_{p}$ is shear stress on the pipe wall; $r_{p}$ is the radius of the pipe section.

Thus, the transfer of motion in solid and liquid media can be described by similar equations.

The equations that are given above describe the movement of an elementary volume of a substance at relatively low speeds of movement. However, in the case of any abrupt changes caused by either external influences or fast-flowing wave processes, there is a need for a deeper study of the process of motion transmission in mechanical systems.

From the study of the phenomelogical Zener model in [13], it was shown that the elastic properties of matter should be described by a generalized function using complex numbers.

In many cases, the imaginary component is substantially less than the real component.

It is known that the interaction of an elastic wave with the interface of media is largely due to the wave resistance, which is determined by the relation [17]:

$-\frac{\sigma}{v}=\rho a_{1}, \quad a_{1}=\Theta\left(\frac{E}{\rho}\right)^{\frac{1}{2}}, \quad \Theta=\left[\frac{(1-\mu)}{(1+\mu)(1-2 \mu)}\right]^{\frac{1}{2}}$,

here $a_{1}$ is velocity of propagation of longitudinal oscillations in the medium; $\mu$ is Poisson's ratio. 
Writing impedance in an operator form, it is possible for $\rho=$ const, $\Theta=1, E_{u}=E_{2}=E$ get:

$Z_{b}(j \omega)=\left[\frac{E_{u}(\omega) \theta(j \omega)}{j \omega}\right], \quad \theta(j \omega)= \pm(j \omega) \sqrt{\frac{\rho}{E}}$

here $\theta(j \omega)$ is operator coefficient of waves propagation. After a one-dimensional transformation of Eqs. (1), (2) according to Laplace with zero initial conditions, we obtain:

$\rho s v(s)=-\frac{d \sigma(s)}{d x}, \frac{E d v(s)}{d x}=-s \sigma(s)$.

The solution of the system of equations under the corresponding boundary conditions [13] is:

$\sigma(x, s)=\sigma_{1}(s, 0) \operatorname{ch}[\theta(s) x]-E v_{1}(s, 0)\left[\frac{\theta(s)}{s}\right] \operatorname{sh}[\theta(s) x]$,
$v(x, s)=v_{1}(s, 0) \operatorname{ch}[\theta(s) x]-\left[\frac{\sigma_{1}(s, 0)}{E}\right]\left[\frac{s}{\theta(s)}\right] \operatorname{sh}[\theta(s) x]$.

For the case of a consistent load (when there are no reflected waves) and $v_{2} \geq 0$ from Eqs. (7), (8) with the boundary conditions $v(s, 0)=v_{1}(s), \quad v(s, l)=v_{2}(s), \quad \sigma(s, 0)=\sigma_{1}(s)$, $\sigma(s, l)=\sigma_{2}(s), \sigma_{2}(s)(1-c)=\left[F(s)+h_{n} v_{2}(s)+m s v_{2}(s)\right] / f_{2}$, where $f_{2}$ is sectional area of the main line in the vicinity of the executive body of mass $m ; c, h_{n}$ is coefficients of friction loss; $F$ is resistance force acting on the executive body; $l$ is length of the force line, we can obtain the equations:

$v_{2}(s)\left[1-c+h_{n} \vartheta_{n}(s) s+m \vartheta_{n}(s) s^{2}\right]=\frac{v_{1}(s)(1-c)}{\operatorname{ch}[\theta(s) l]}-F(s) \vartheta_{n}(s) s$,

here $\vartheta_{n}(s)=\vartheta_{n 0} Z_{n}(s) ; \vartheta_{n 0}=l /\left(E f_{2}\right) ; Z_{n}(s)=t h A / A ; A=\theta(s) l$.

The equation describing the dynamics of a volumetric hydraulic drive, after similar transformations, is written in the form $[18,19]$ :

$$
\begin{aligned}
& \Omega(s)\left\{s^{2} J \vartheta_{s}(s)+s\left[J \tau+\vartheta_{s}(s) h\right]+1-c+\tau h\right\}=Q(s)(1-c) \Psi(s) \\
& \quad-M_{r}(s)\left[\vartheta_{s}(s) s+\tau_{k}\right],
\end{aligned}
$$

where:

$\vartheta_{s}(s)=\frac{\vartheta_{s 0} Z_{n}(s)}{E_{g}}, \quad \vartheta_{s 0}=\frac{\left(l f_{1}\right)}{\kappa_{1} w^{2}}, \quad \tau=\frac{K_{1}}{w^{2}}, \quad Z(s)=\frac{t h A_{1}}{A_{1}}, \quad A_{1}=\theta_{01}(s) L, \theta_{01}(s)=\left(\frac{\rho_{0}}{E}\right)^{\frac{1}{2}}$.

In Eq. (8), the notation: $\tau$ is hydraulic tightness criterion; $\vartheta_{0 s}$ is coefficient of elasticity; $E_{g}(s)$, $\Psi(s)$ is polynomials obtained during transformations; $w$ is volumetric constant of the hydraulic motor; $Q$ is pump flow rate; $\theta_{01}(s)$ is operator coefficient of wave propagation in the pressure line; $J$ is moment of inertia of rotating parts; $c$ is coefficient of friction loss proportional to the pressure drop; $h$ is coefficient of friction loss proportional to the velocity of motion; $\rho_{0}, \kappa$ is density and bulk modulus of elasticity of the liquid; $\Omega$ is frequency of rotation of the output shaft; $M_{r}$ is moment of resistance; $K_{l}$ is fluid leakage rate.

Wherein, the boundary conditions were determined from the balance of the flow of fluid entering the pipelines and flowing from them. 


\section{Stability of open two-link systems with distributed parameters}

In the study of stability Lyapunov [3] used two methods. 1) Covers the methods that lead to the definition of the general and particular solutions of differential equations. 2) Without finding solutions to differential equations, it is required to find a function of generalized coordinates and time, the total derivative of which, by virtue of the equations of motion under consideration, has certain properties (Lyapunov function).

The development of the second method, as the most convenient, led to the use of linearized differential equations with constant coefficients and of special stability criteria proposed by Routh, Gurwitz, Mikhailov, Nyquist, etc. Referring to Eqs. (12), (10), we see that the coefficients of the characteristic equations with variable s depend on the same variable. Since the use of these criteria turned out to be difficult, a new technique for assessing sustainability was developed using the first Lyapunov method. From Eqs. (8), (10), it can be seen that the dynamic properties of the systems significantly depend on the functions $\vartheta_{n}(s), \vartheta_{s}(s)$. If we consider the oscillations in the system, then such a function, in addition to density, elastic modulus (shear) and line geometry, depends on the oscillation frequency. At the same time for longitudinal vibrations in the rod can be written [14]:

$Z_{k}(j \omega)=\frac{\operatorname{th}\left[\theta_{k}(j \omega) l\right]}{\theta_{k}(j \omega) l}=\frac{j \operatorname{tg} \alpha}{j \alpha}=\frac{\operatorname{tg} \alpha}{\alpha}, \alpha=\omega l \sqrt{\frac{\rho}{G}}, \operatorname{ch}(j \alpha)=\cos \alpha$,

here $\alpha$ is a real dimensionless quantity. Therefore, $\vartheta_{n}(s)=\vartheta_{n}(\alpha)$ and $\cos (\alpha)$ are not complex functions. These considerations are valid and for torsional vibrations.

From the analysis of the function $Z_{n}(\alpha)$ follows: with $\alpha \rightarrow 0, Z_{n} \rightarrow 1$; for:

$\frac{\pi}{2}+k \pi>\alpha>\pi+k \pi, Z_{n}<0$

here $k=0,1,2, \ldots, n$.

Analyzing Eqs. (8), (10), we see that they are similar. In this regard, we will carry out the following reasoning with the equation for the volumetric hydraulic drive Eqs. (10), with which it was easier to perform an oscillographic recording of the oscillatory process in natural experiments. After the algebraic decomposition of Eq. (10) into two equations and the inverse transition from the Laplace images to the originals, we obtain the equations:

$$
\begin{aligned}
& Q(t) \Psi(\omega)=w \Omega(t)+\tau w^{2} p(t)+\vartheta(\alpha) w^{2}\left(\frac{d p}{d t}\right), \\
& p(t) w(1-c)=M_{r}(t)+h \Omega(t)+J\left(\frac{d \Omega}{d t}\right),
\end{aligned}
$$

here $p$ is the differential pressure on the hydraulic motor. Wherein, the complex functions $\Psi(s)$ and $E(s)$ in (10) are replaced by their real parts $\Psi(\omega)$ and $\vartheta(\alpha)=\vartheta_{0} E^{-1}(\omega) Z(\alpha)$, which substantially exceed modulo imaginary parts $[15,19]$. The admissibility of using Eq. (12) to study the dynamic properties of the systems under consideration was checked by constructing the frequency characteristics of a volumetric hydraulic drive in various ways [13]: calculating the frequency characteristics of the 4th order upgraded Runge-Kutta method $[20,21]$ and from a natural experiment.

Next, we introduce the operator $D \equiv d / d t$, and with $M_{r}=$ const we transform the system Eq. (12):

$k Q(t)=\frac{\tau M_{r}(t)}{1-c+\tau h}+\Omega(t)\left(1+2 \zeta T D+T^{2} D^{2}\right)$, 
where:

$2 \zeta T=\frac{[J \tau+h \vartheta(\alpha)]}{1-c+\tau h}, \quad T=\left[\frac{j \vartheta(\alpha)}{1-c+\tau h}\right]^{\frac{1}{2}}, \quad \zeta=0,5\left[\frac{\tau}{\vartheta(\alpha)}+\frac{h}{j}\right], \quad \kappa=\left[\frac{1-c}{(1-c+\tau h) w}\right] \Psi(\omega)$.

We assume that the ratio $\Omega_{n}=\Omega+\frac{\tau M_{r}}{1-c+\tau h}$ is the reduced velocity, and $\left(\tau M_{r}\right) /(1-c+\tau h)$ is the static error. Then Eq. (13) can be rewritten:

$k Q(t)=\Omega_{n}(t)\left(1+2 \zeta T D+T^{2} D^{2}\right)$.

Eq. (14) describes the dynamics of the system in deviations from a certain static mode at $Q(t)=Q_{0}$ and the moment of resistance $M_{r}(t)=M_{r 0}$ with a steady rotation frequency $\Omega=\Omega_{0}$.

Due to the non-uniformity of friction in the pistons of the axial-piston hydraulic motor, we assume that the moment of resistance oscillates with a small amplitude according to the law:

$M_{r}(t)=M_{r 0}\left[1+b \sin \left(a \Omega_{0} t\right]\right.$.

In expression (15), the introduced factor " $a$ " takes into account the possibility of generating oscillations from the other pistons of the hydraulic motor. Consequently, at a known speed in the linear system there will be stable small oscillations with a frequency $\omega_{0}=a \Omega_{0}$. At such a frequency, the coefficients $\Psi(\omega), \vartheta(\alpha)$ will have quite definite values $\Psi\left(\omega_{0}\right), \vartheta\left(\alpha_{0}\right)$.

We rewrite Eq. (15) in Laplace images for the case when there are no deviations from the steady state operation in the system, and define the transfer function:

$W=\frac{\Omega_{n}(s)}{Q(s)}=\frac{k}{\left(1+2 \zeta T s+T^{2} s^{2}\right)}$.

With a small jump action $\Delta Q(t)=q_{0} \cdot 1(t)$, when changes of the coefficients can be neglected, the equation of motion in the images will look like:

$\Omega_{n}(s)\left(1+2 \zeta T s+T^{2} s^{2}\right)=\frac{k q_{0}}{s}$.

Using transition tables [13] from images to originals, we obtain:

$\Omega_{n}(t)=k\left\{1-\exp (-\alpha t)\left[\cos (\omega t)+\frac{\alpha \sin (\omega t)}{\omega}\right]\right\}$,

where:

$\alpha=\frac{\zeta}{T}=0,5\left[\frac{\tau}{\vartheta_{h}(\alpha)}+\frac{h_{h}}{j}\right], \quad \omega=\left[\frac{1-\zeta^{2}}{T^{2}}\right]^{\frac{1}{2}}, T=\left[\frac{j \vartheta_{h}(\alpha)}{1-c+\tau h_{h}}\right]^{\frac{1}{2}}$.

From Eq. (18) it can be seen that when $\alpha>0$, and therefore $\vartheta(\alpha)>0$, the value of $\Omega_{n}$ tends to some steady value, that is, the process is stable. If $\vartheta(\alpha)<0$ and $\alpha<0$, then the quantity $\Omega_{n}$ tends to infinity, i.e. the process will be unstable. Let now the hydraulic drive switched to another $\Omega=\Omega_{01}$. In this case, the coefficients of Eq. (17) will have other values and the response to a small change in the impact will be different.

If, at the same pump feed, the new value of the resistance moment due to leakage causes the output motor shaft to rotate with another steady frequency $\Omega_{01}$, then the coefficients of 
characteristic Eq. (17) and transfer function $W(s)$ will also be different which will be determined by the steady state frequency $\Omega_{01}$.

It was shown above that the values of the functional coefficients in the equations depend on the functions $Z(\alpha), Z_{n}(\alpha), Z_{k}(\alpha)$. From their analysis it can be seen that, periodically, $Z$ becomes negative. Since, depending on the pump flow or static load, the stationary frequency of rotation of the motor will be different, it is possible that, under certain operating conditions, the coefficients of the characteristic Eq. (17) become negative. In this case, with a small discontinuous change in the disturbing influence, the rotation frequency will either increase indefinitely or decrease to zero, i.e. the system will be unstable. Thus, the stability of an open-loop system is determined by the inequality $\operatorname{tg} \alpha / \alpha \geq 0$, where $\alpha=l \omega(\rho / \chi)^{1 / 2} ; \chi$ is the coefficient of elasticity the material.

In nonlinear systems, for example, in drilling hydraulic drives, loss of stability leads to auto-oscillations.

\section{Conclusions}

The study showed that open two-link systems, depending on the frequency of disturbing oscillations and the properties of the lines of force, may become unstable. Loss of stability will consist either in stopping the work or in the appearance of self-oscillations.

\section{References}

[1] Routh E. J. The Advanced Part of a Treatise on the Dynamics of a System of Rigid Bodies. Macmillan and Co, London, 1884.

[2] Hurwitz A. Ueber die Bedingungen, unter welchen eine Gleichung nur Wurzeln mit negativen reellen Teilen besitzt. Mathematische Annalen, Vol. 46, 1895, p. 273-284.

[3] Lyapunov A. M. The General Problem of the Stability of Motion. Taylor and Francis, London, 1992.

[4] Chetaev N. G. Stability of Motion. Gostekhizdat, Moscow, 1950.

[5] Nyquist H. Regeneration theory. Bell System Technical Journal, Vol. 11, Issue 1, 1932, p. 126-147.

[6] Popov E. P. Applied Theory of Control Processes in Nonlinear Systems. Nauka, Moscow, 1973.

[7] Berezyansky Yu M., Kondrat'ev Yu G. Spectral Methods in Infinite-Dimensional Analysis. ANUSSR, Naukova Dumka, Kiev, 1988.

[8] Godunov S. K., Ryaben'kij V. S. Difference Schemes. FML, Nauka, Moscow, 1977.

[9] Encyclopedia. Vols. 1-4, Mashinostroenie, Moscow, 2000.

[10] Sirazetdinov T. K. Stability of Systems with Distributed Parameters. Nauka, Novosibirsk, 1987.

[11] Sedov L. I. Continuum Mechanics. Vol. 1, 2, Nedra, Moscow, 1970.

[12] Postnikov V. S. Internal Friction in Metals. Metallurgiya, Moscow, 1974.

[13] Kondratenko L. A. Vibrations and Speed Regulation Methods of Movement of Technological Objects. Moscow, MRSU, 2005.

[14] Kondratenko L., Mironova L. Features of loss of stability of the work of two-link mechanisms that have an infinite number of degrees of freedom. International Journal of Mathematical, Engineering and Management Sciences, Vol. 3, Issue 4, 2018, p. 315-334.

[15] Kondratenko L., Mironova L., Terekhov V. Investigation of vibrations during deepholes machining. Journal of Vibroengineering Procedia, Vol. 11, 2017, p. 7-11.

[16] Popov D. N. Dynamics and Regulation of Hydro-Pneumatic Systems. Mashinostroenie, Moscow, 1995.

[17] Physical Encyclopedia. Vol. 4, Bol'shaya Rossijskaya, 1994.

[18] Kondratenko L. A., Mironova L. I. Imitation of nonlinear drives with distributed parameters of power lines. Engineering and Automation Problems, Vol. 1, 2018, p. 92-97.

[19] Kondratenko L., Dmitriev V., Mironova L. Simulation of a drive with a long connecting link. Journal of Vibroengineering Procedia, Vol. 12, 2017, p. 231-236.

[20] Kamke E. Handbook of Ordinary Differential Equations. Leipzig, 1959.

[21] Kondratenko L. A., Mironova L. I. Simulation Model of a Mechanical System with Significant Non-Linearities "GDPR1_5". Certificate of State Registration of Computer Programs No. 2018617684, 2018. 